\title{
Neumoconiosis asociada a la exposición de polvo de carbón y de sílice en sitios de minería subterránea en el departamento del Cauca: presentación de 3 casos
}

\author{
Pneumoconiosis associated with coal dust and \\ silica exposure in underground mines in Cauca \\ Departament: presentation of 3 cases
}

Juan David Orozco B. ${ }^{1}$, Hernando David Vargas S. ${ }^{1}$, Julia Edith Chamorro. ${ }^{2}$

\section{Resumen}

INTRODUCCIÓN: la neumoconiosis asociada a la exposición de polvos en minas subterráneas, como el carbón y la sílice, continúa siendo un problema de salud debido al compromiso pulmonar irreversible que impacta en la calidad de vida de las personas expuestas a estos agentes, para los cuales solo existe el manejo sintomático. No existen casos previos reportados en el departamento del Cauca, lo cual motiva a la presentación de esta serie de casos.

DESCRIPCIÓN DE CASOS: se trata de 3 pacientes con un cuadro de disnea y tos crónica con historia de trabajo en una minería subterránea. En la tomografía axial computarizada de alta resolución (TACAR) de tórax se encontró un patrón característico de neumoconiosis.

DISCUSIÓN: los 3 pacientes tienen una historia importante de exposición a minas subterráneas, con hallazgos imagenológicos similares. Sin embargo, algunas características clínicas difieren de la población estudiada en el ámbito mundial.

Palabras clave: neumoconiosis, minería, enfermedad pulmonar intersticial.

\begin{abstract}
INTRODUCTION: Pneumoconiosis associated with dust exposure in underground mines, such as coal and silica, continues to be a health problem, due to irreversible lung compromise, which affects the quality of life of people exposed to these agents, for whom only exist a symptomatic treatment. There aren't cases reported in Cauca Department, which motivates the presentation of these cases.
\end{abstract}

CASE DESCRIPTION: These are 3 patients with symptoms of dyspnea and chronic cough with a history of work in underground mines, with findings of a suggestive pattern of pneumoconiosis in the high-resolution computed tomography of the chest.

DISCUSSION: All 3 patients have significant history of exposure to underground mines, with similar findings in the image. However, some clinical characteristics differ from the worldwide studied population.

Keywords: Pneumoconiosis; Mining; Interstitial Lung Disease.
${ }^{1}$ Residente de Medicina Interna, Universidad del Cauca. Popayán, Colombia.

${ }^{2}$ Internista, neumóloga y docente del Departamento de Medicina Interna, Universidad del Cauca. Popayán, Colombia

Autor de correspondencia

Oscar Sáenz M.

Correo electrónico:

jdorozco@unicauca.edu.co

Recibido: 20/10/2020

Aceptado: 28/11/2020 


\section{Introducción}

La minería continúa siendo una de las industrias con gran importancia en el desarrollo económico. En Colombia esta actividad genera un $2,1 \%$ del producto interno bruto (PIB) (1); sin embargo, existen zonas donde no está regulada su actividad, lo cual genera una exposición importante y continua derivada de la falta de elementos de protección necesarios para el desarrollo de la labor de campo. A las enfermedades relacionadas con la inhalación de un agente en la minería se les denomina neumoconiosis (2). Dentro de la exposición minera se destacan dos componentes: carbón y sílice, para los cuales se ha descrito un compromiso intersticial importante, con características clínicas y en las imágenes similares (3). No obstante, para el desarrollo de este compromiso no solo se requiere de la exposición, sino de otros factores como la concentración y un tiempo determinado de exposición $(4,5)$.

La prevalencia a nivel mundial de esta enfermedad es variable y puede ir desde $0,8 \%$ hasta $6,02 \%$ (6); para Colombia se estima un $5 \%$ de prevalencia (7). Estos datos dependen de la población estudiada y del tiempo de exposición al agente, siendo más frecuente en mineros con edad comprendida entre 50-60 años y con más de 25 años de exposición $(8,9)$. Por otra parte, se calcula que la neumoconiosis derivada de la exposición a la sílice, el asbesto y el polvo de carbón ocasiona 30000 muertes al año y 1240000 años de vida saludables perdidos (10).

El diagnóstico, sumado a la historia de exposición, se basa en los hallazgos en las imágenes; inicialmente con una radiografía del torax o según la disponibilidad y hallazgos una TACAR del tórax (11). En pocos casos se requiere de la biopsia pulmonar, sobre todo cuando el diagnóstico por imagen es incierto o se busca descartar otras etiologías (12). Finalmente se reitera que en el departamento del Cauca no hay una descripción de casos con esta patología, lo cual motiva esta publicación académica.

\section{Presentación de casos}

\section{Caso 1}

Paciente masculino de 45 años con un cuadro clínico de 3 meses de evolución caracterizado por tos seca persistente, ocasionalmente con expectoración hialina, sin otros síntomas asociados. Tenía antecedentes de tabaquismo con un índice de paquete año (IPA) no estipulado; además, historia de trabajo en una minería con exposición al carbón y a la sílice sin la debida protección durante 10 años.

Se manejó inicialmente como una enfermedad obstructiva crónica (EPOC) con agonistas $\beta 2$ y anticolinérgicos sin respuesta, por lo cual se derivó a neumología. En el examen físico de su primera consulta tenía los siguientes signos vitales: presión arterial (PA) de 116/85 mm Hg, frecuencia cardíaca (FC) de 67 latidos por minuto (lpm), frecuencia respiratoria (FR) de 18 respiraciones por minuto (rpm), saturación de oxígeno $\left(\mathrm{SatO}_{2}\right)$ al aire ambiente de $94 \%$ y un peso de $60 \mathrm{~kg}$. A nivel físico, como hallazgos positivos, se encontró un soplo tubárico apical bilateral.

Por la historia y los antecedentes descritos se realizó una espirometría, una TACAR de tórax y estudios para descartar tuberculosis (TBC). En la espirometría se documentó una alteración ventilatoria obstructiva moderada que no se modificaba con el $\beta 2$ inhalado con capacidad vital forzada (CVF) de 3,59 (93\%), volumen espirado en el primer segundo $\left(\mathrm{VEF}_{1}\right)$ de 2,14 (66 $\%$ ) relación del $71 \%$ con un volumen espirado forzado (FEF) $25 \%$-75 \% de 1,03 (28\%). La TACAR de tórax presentaba hiperdensidades intersticiales difusas de tipo micronodular, con imágenes de adenopatías hiliares y parahiliares bilaterales. Los estudios de TBC fueron negativos. Por lo anterior, se realizó el diagnóstico de neumoconiosis y EPOC, y el paciente recibió manejo con fluticasona/vilanterol 22/92 $\mu \mathrm{g}$ en 1 inhalación cada 24 horas y umeclidinio $55 \mu \mathrm{g}$ en 1 inhalación cada 24 horas, con lo que se obtuvo una mejoría sintomática y de su patrón obstructivo medido por medio de una espirometría de control.

\section{Caso 2}

Paciente masculino de 74 años con un cuadro clínico de 4 años de evolución, caracterizado por tos seca persistente, asociado a una expectoración blanquecina, con síntomas constitucionales como pérdida de peso no cuantificada. Como único antecedente se destaca la historia de trabajo en una minería con exposición al carbón y a la sílice sin la debida protección durante 9 
años. Además, historia de múltiples hospitalizaciones por cuadros infecciosos.

Tenía imágenes radiográficas que describían la presencia de opacidades intersticiales reticulares ampliamente distribuidas en ambos hemitórax (Figura 1), por lo cual fue enviado a valoración por neumología. En su primera valoración, en el examen físico, presentaba los siguientes hallazgos (signos vitales): PA de 104/62 mm $\mathrm{Hg}, \mathrm{FC}$ de $81 \mathrm{lpm}$, FR de $20 \mathrm{rpm}, \mathrm{SatO}_{2}$ de $93 \%$ con una fracción inspirada de oxígeno $\left(\mathrm{FIO}_{2}\right)$ del $28 \%$ y un peso de $50 \mathrm{~kg}$. En el examen, como hallazgo positivo a nivel pulmonar, se destacaba la presencia de estertores finos bilaterales sin otros ruidos sobreagregados.

Se ampliaron estudios; en la TACAR de tórax presentaba opacidades mixtas bilaterales con un componente intersticial, alveolar reticular y micronodular. En la espirometría se documentó una alteración restrictiva severa con un componente obstructivo severo de la vía aérea periférica que no se modifica con el $\beta 2$ inhalado con una CVF de 1,5 L (40 \%), $\mathrm{VEF}_{1}$ de 1,11 L (38 \%), FEF $25 \%$-75 \% de $760 \mathrm{~mL}$ (27\%). Además, el paciente tenía estudios para TBC negativos. Ante estos hallazgos se diagnostica una enfermedad pulmonar intersticial difusa severa asociada a neumoconiosis. Dado el estado avanzado de su enfermedad se le da manejo con indacaterol y glicopirronio 110/50 microgramos $(\mu \mathrm{g})$ en 1 inhalación cada 24 horas y oxigenoterapia.

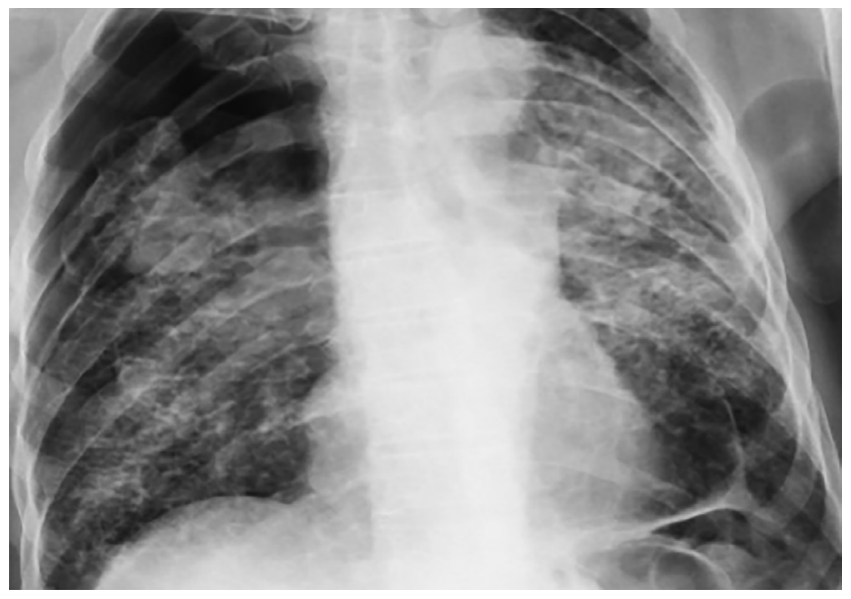

Figura 1. Radiografía de tórax: opacidades intersticiales reticulares grandes, ampliamente distribuidas en ambos hemitórax $(D, I)$, sin anormalidades pleurales u obliteración del ángulo costofrénico (según la Organización Internacional del Trabajo [OIT]).

\section{Caso 3}

Paciente masculino de 45 años con un cuadro clínico de 8 meses de evolución, caracterizado por la presencia de dolor torácico en la región paraesternal (tipo punzada) asociado a disnea de moderados esfuerzos. Además, en ocasiones relataba la presencia de fiebre subjetiva no cuantificada sin otro tipo de síntomas descritos.

Consultó a urgencias en distintas ocasiones, donde se descartaba una etiología cardiovascular con electrocardiograma y enzimas cardíacas negativas. Previa valoración por neumología, consulta por una exacerbación de su cuadro a urgencias, donde le realizan una radiografía del tórax, en la que describen la presencia de una consolidación en el segmento apical derecho; también le realizan una prueba de dímero $\mathrm{D}$, la cual es negativa. Por estos hallazgos se inicia un manejo antibiótico y una valoración ambulatoria por neumología. Como único antecedente presenta una historia de trabajo en minería con exposición al carbón y a la sílice sin la debida protección durante 20 años.

En el examen físico de su primera valoración tenía los siguientes signos vitales: PA 135/79 mm Hg, FC de $87 \mathrm{lpm}, \mathrm{FR}$ de $18 \mathrm{rpm}, \mathrm{SatO}_{2}$ al aire ambiente de $92 \%$ y un peso de $57 \mathrm{~kg}$. A nivel físico se destacaba la presencia de escasos estertores bibasales. Por lo previamente mencionado se ampliaron estudios, entre ellos, TACAR de tórax, en el que se encontraron consolidaciones posteriores bilaterales e infiltrado micronodular de predominio en los segmentos superiores del lóbulo superior derecho (Figura 2). Por la historia de trabajo en minería se solicitó una fibrobroncoscopia más lavado broncoalveolar con estudios, los cuales descartaron una etiología infecciosa, entre ellos TBC e histoplasmosis. La espirometría presentaba una alteración obstructiva leve que no se modifica con el $\beta 2$ inhalado con CVF de 3,52 (84 \%) $\mathrm{VEF}_{1}$ de 2,47 (74\%), una relación de $89 \%$ y un FEF $25 \%-75 \%$ de 1,76 (56 \%).

\section{Discusión}

La neumoconiosis sigue siendo una patología con gran impacto en la población afectada dadas las complicaciones derivadas de un marcado compromiso pulmonar, y no existe un manejo específico. Cabe resaltar que para el departamento del Cauca no existen datos 
sobre esta patología, y según la Agencia Nacional de Minería de Colombia no existe un proyecto minero, lo que alerta sobre la existencia de un problema de salud pública no reconocido.

De estos pacientes, todos tenían un tiempo de exposición $<25$ años, lo que contrasta con lo establecido en estudios previos para el desarrollo de esta patología $(9,10)$. Además, en la espirometría predominó un patrón obstructivo, el cual se equipara con lo reportado por Romero y colaboradores (8). En torno al diagnóstico, este se realizó reuniendo el principal factor de riesgo (exposición) junto con hallazgos en las imágenes y las radiografías del tórax guiadas por la clasificación de la OIT, la cual determina una puntuación por medio de opacidades grandes (dimensión >10 mm), pequeñas, anormalidades pleurales (se registran como presentes si tienen un ancho $>3 \mathrm{~mm}$ ), obliteración del ángulo costofrénico (presente o ausente) y otros hallazgos (11) (Tabla 1), o hallazgos en la TACAR de tórax que se caracterizan por la presencia de pequeños nódulos redondeados localizados predominantemente en zonas superiores del pulmón, con diversos grados de adenopatía mediastínica que puede estar o no calcificada (12-14). Aunque la TACAR de tórax presenta una mayor sensibilidad que la radiografía simple, la primera no se suele emplear como estudio inicial dada la limitada disponibilidad, el grado de radiación y las dificultades en la interpretación por parte de personal no entrenado (13). Ninguno de los pacientes requirió la realización de una biopsia por la historia y los hallazgos imagenológicos.
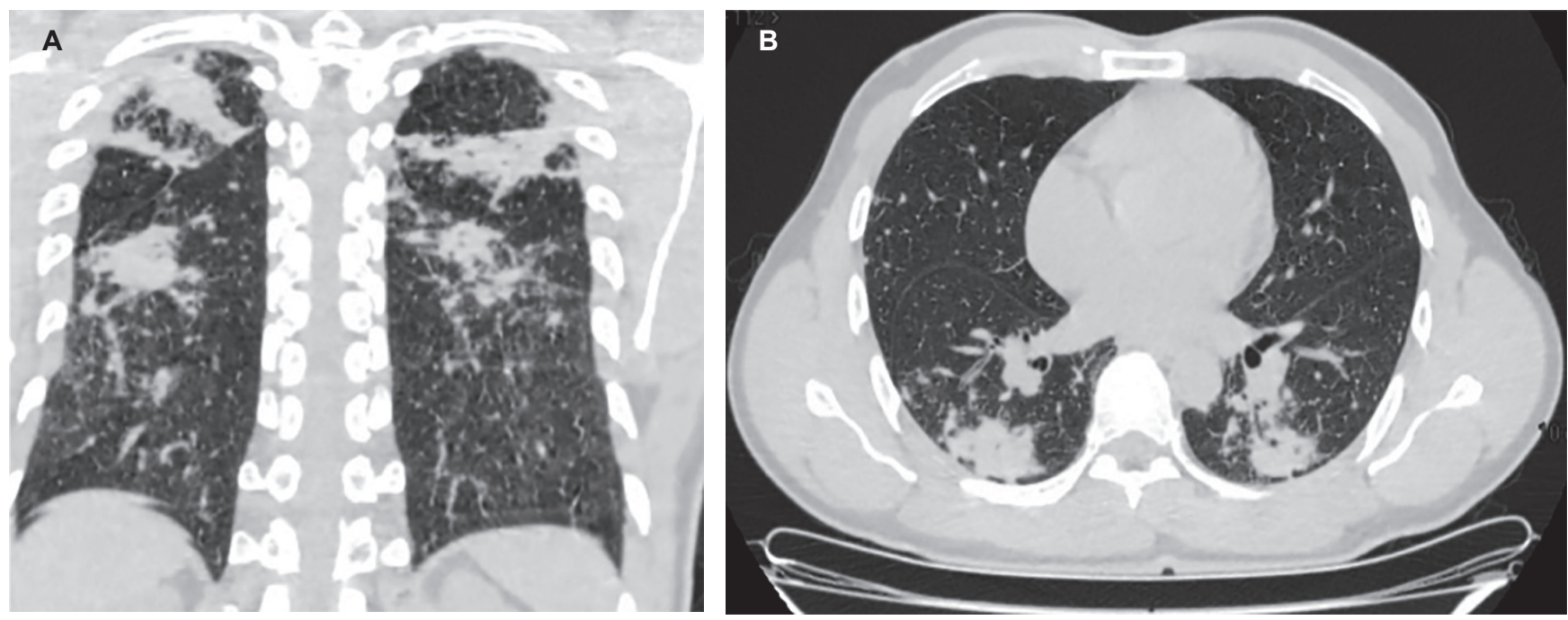

Figura 2. A. Corte sagital de TACAR de tórax con consolidaciones posteriores bilaterales e infiltrado micronodular de predominio en los segmentos superiores del lóbulo superior derecho. B. Corte axial con hallazgos similares descritos. 
Tabla 1. Clasificación de la OIT para el diagnóstico de neumoconiosis en rayos $\mathrm{X}$ de tórax. Adaptado de la referencia 11.

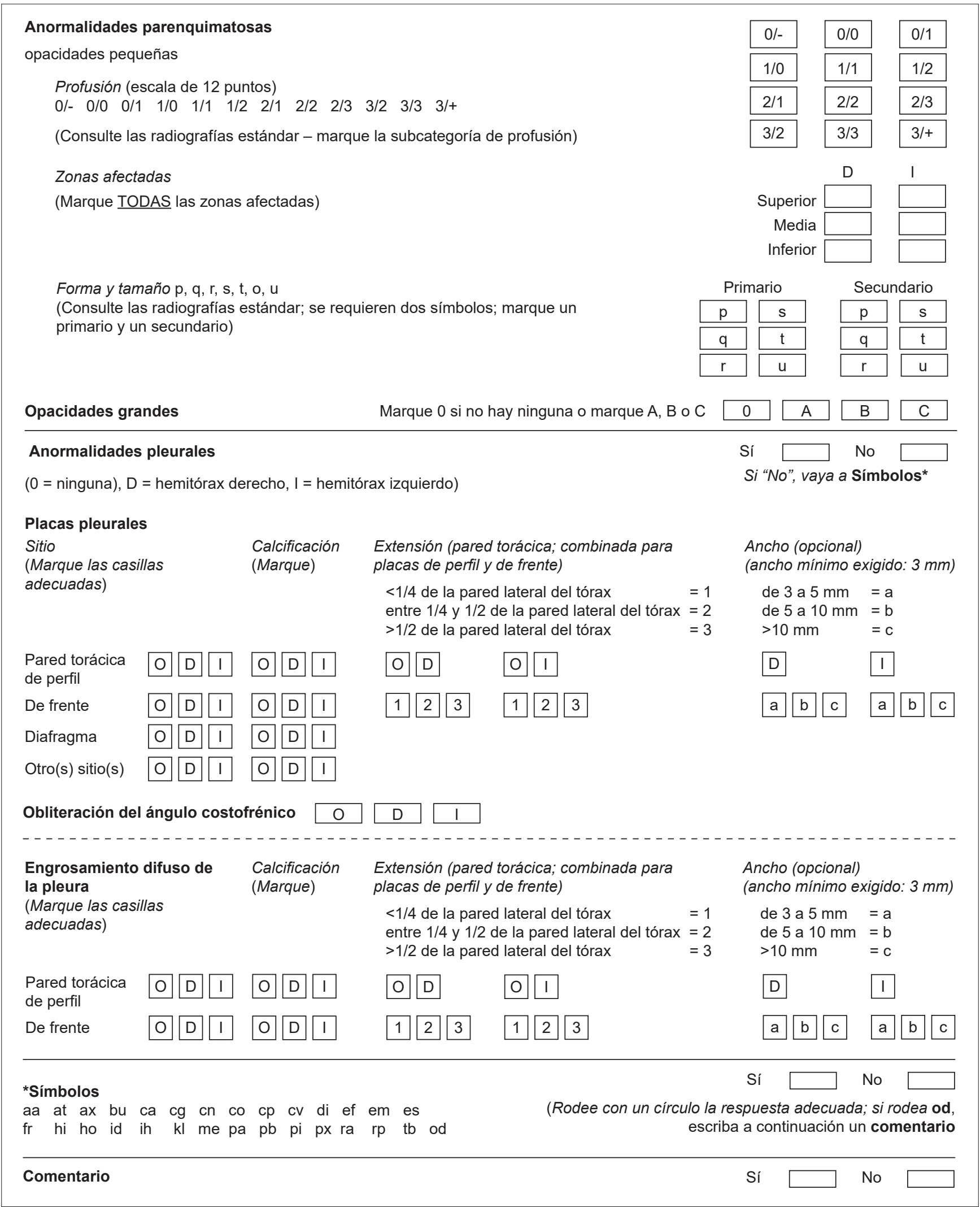




\section{Conclusiones}

Este es el primer reporte de casos realizado en el departamento del Cauca. Los 3 casos descritos, en algunas características clínicas, difieren con los datos publicados en reportes previos, lo cual motiva a la realización de estudios ulteriores que complementen y visualicen la situación actualmente mencionada.

\section{Referencias}

1. Portafolio [Internet]. Bogotá: Portafolio; 25 de febrero de 2019. Tovar Martínez E. Minería bien hecha, una 'joya' para la economía colombiana. Disponible en: https://www.portafolio.co/economia/mineria-bien-hecha-una-joya-para-la-colombiana-526780

2. Fishwick D, Barber C. Pneumoconiosis. Medicine. 2012;40(6):310-13. doi: 10.1016/j.mpmed.2012.03.011

3. Perlman DM, Maier LA. Occupational Lung Disease. Med Clin North Am. 2019;103(3):535-48. doi: 10.1016/j. mena.2018.12.012

4. Ministerio de Protección Social de Colombia [Internet]. Guía de Atención Integral Basada en la Evidencia para Neumoconiosis (Silicosis, Neumoconiosis del minero de carbón y Asbestosis) (GATI-NEUMO); 2006. Disponible en: https:// www.epssura.com/guias/gati neumo.pdf

5. Ordúz E. Guías de la Asociación Colombiana de Neumología y Cirugía del Tórax-Neumología Ocupacional. Rev Colomb Neumol. 2009;21;37-43.

6. Mo J, Wang L, Au W, Su M. Prevalence of coal workers' pneumoconiosis in China: a systematic analysis of 2001-2011 studies. Int J Hyg Environ Health. 2014;217(1):46-51. doi: 10.1016/j.ijheh.2013.03.006
7. Mazuera M, Grisales H, Rendón I. Neumoconiosis en la minería subterránea del carbón, Amaga, 1995. Rev. Fac. Nac. Salud Pública. 1997;14(2):46-67.

8. Romero M, Varona M, Pinilla M, Briceño L. Prevalence of pneumoconiosis and spirometric findings in underground mining workers in Cundinamarca, Colombia. Rev. Fac. Med. 2019;67(4):581-86. doi: 10.15446/revfacmed.v67n4.72201

9. Driscoll T, Nelson DI, Steenland K, Leigh J, Concha-Barrientos M, Fingerhut M, et al. The global burden of non-malignant respiratory disease due to occupational airborne exposures. Am J Ind Med. 2005;48(6):432-45. doi: 10.1002/ajim.20210

10. Page SJ, Organiscak JA. Suggestion of a cause-and-effect relationship among coal rank, airborne dust, and incidence of workers' pneumoconiosis. AIHAJ. 2000;61(6):785-7.

11. Oficina Internacional del Trabajo [Internet]. Guía para el uso de la clasificación internacional de la OIT de radiografías de neumoconiosis. Ginebra: OIT; 2011 [falta fecha de acceso]. Disponible en: https:/www.ilo.org/wcmsp5/groups/public/--ed_protect/---protrav/---safework/documents/publication/ wcms_223941.pdf

12. Kalchiem-Dekel O, Galvin JR, Burke AP, Atamas SP, Todd NW. Interstitial Lung Disease and Pulmonary Fibrosis: A Practical Approach for General Medicine Physicians with Focus on the Medical History. J Clin Med. 2018;7(12):476. doi: $10.3390 / \mathrm{jcm} 7120476$

13. Corrin B, Nicholson AG. Occupational, environmental and iatrogenic lung disease. Pathology of the Lungs. 2011;327-99. doi: 10.1016/B978-0-7020-3369-8.00007-0

14. Novo Rivas U, Varela Modroño JL, Romero Cique F, Toranzo Ferreras P, Morgade Diéguez MI. Cómo realizar un informe radiológico de neumoconiosis según radiografías estándar digitalizadas de la ILO. SERAM. 2014;1-19. doi: 10.1594/ seram2014/S-0738 
